\title{
Self management programme for ankylosing spondylitis (Protocol)
}

\author{
Spencer S, Martindale JH, MacPhie E, Montgomery P
}

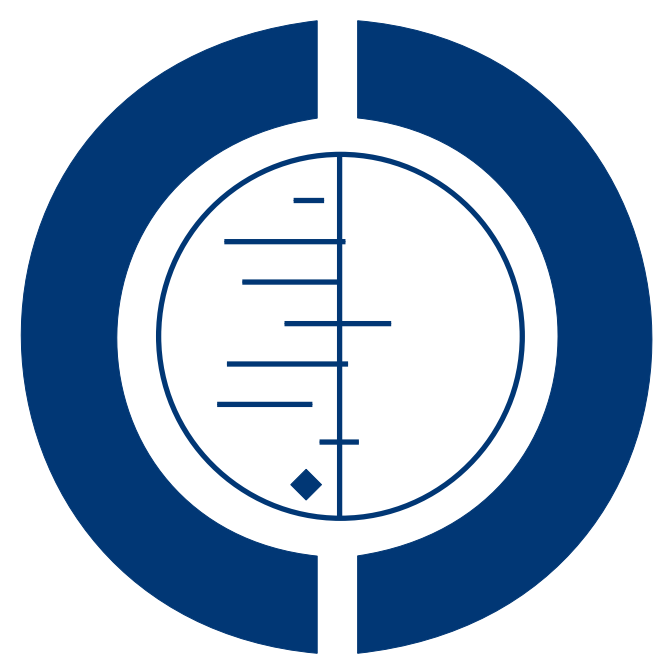

\section{THE COCHRANE COLLABORATION $^{\circledR}$}

This is a reprint of a Cochrane protocol, prepared and maintained by The Cochrane Collaboration and published in The Cochrane Library 2015, Issue 12

http://www.thecochranelibrary.com

\section{WILEY}


TABLE OF CONTENTS

HEADER . . . . . . . . . . . . . . . . . . . . . . . . . . . . . . . . . . . . 1

ABSTRACT . . . . . . . . . . . . . . . . . . . . . . . . . . . . . . . . . . . . . . . . . .

BACKGROUND . . . . . . . . . . . . . . . . . . . . . . . . . . . . . . . . . . . .

OBJECTIVES . . . . . . . . . . . . . . . . . . . . . . . . . . . . . . . . . . . . . . . . . .

METHODS . . . . . . . . . . . . . . . . . . . . . . . . . . . . . . . . . . . . . .

ACKNOWLEDGEMENTS . . . . . . . . . . . . . . . . . . . . . . . . . . . . . . . . . . . . . . . .

REFERENCES . . . . . . . . . . . . . . . . . . . . . . . . . . . . . . . . . . . . . 7

APPENDICES . . . . . . . . . . . . . . . . . . . . . . . . . . . . . . . . . . . . . 10

WHAT'S NEW . . . . . . . . . . . . . . . . . . . . . . . . . . . . . . . . . . . . . . 11

HISTORY . . . . . . . . . . . . . . . . . . . . . . . . . . . . . . . . . . . . . . . . . . 11

CONTRIBUTIONS OF AUTHORS . . . . . . . . . . . . . . . . . . . . . . . . . . . . . . . . . . . . .

DECLARATIONS OF INTEREST . . . . . . . . . . . . . . . . . . . . . . . . . . . . . . 11 


\title{
[Intervention Protocol]
}

\section{Self management programme for ankylosing spondylitis}

\author{
Sally Spencer ${ }^{1}$, Jane H Martindale ${ }^{2}$, Elizabeth MacPhie ${ }^{3}$, Paul Montgomery ${ }^{4}$ \\ ${ }^{1}$ Faculty of Health and Social Care, Edge Hill University, Ormskirk, UK. ${ }^{2}$ Physiotherapy, Wrightington, Wigan and Leigh NHS \\ Foundation Trust, Wigan, UK. ${ }^{3}$ Rheumatology Department, Lancashire Care NHS Foundation Trust, Preston, UK. ${ }^{4}$ Department of \\ Social Policy and Social Work, University of Oxford, Oxford, UK
}

Contact address: Sally Spencer, Faculty of Health and Social Care, Edge Hill University, St Helens Road, Ormskirk, Lancashire, L39 4QP, UK. spencesa@edgehill.ac.uk.

Editorial group: Cochrane Musculoskeletal Group.

Publication status and date: Edited (no change to conclusions), published in Issue 12, 2015.

Citation: Spencer S, Martindale JH, MacPhie E, Montgomery P. Self management programme for ankylosing spondylitis. Cochrane Database of Systematic Reviews 2015, Issue 12. Art. No.: CD006977. DOI: 10.1002/14651858.CD006977.pub2.

Copyright (C) 2015 The Cochrane Collaboration. Published by John Wiley \& Sons, Ltd.

\begin{abstract}
A B S T R A C T
This is the protocol for a review and there is no abstract. The objectives are as follows:

To assess the benefits and harms of self management programmes for people with AS/Axial SpA.
\end{abstract}

\section{B A C K G R O U N D}

\section{Description of the condition}

Ankylosing spondylitis (AS) is a chronic systemic inflammatory musculoskeletal condition belonging to a family of diseases known as the spondyloarthropathies (Khan 2003). This condition is characterised by inflammation of spinal joints and adjacent structures that may lead to progressive and ascending bony fusion of the spine (Davis 2005). The exact origin of AS is unknown, but it is recognised that both environmental and genetic factors play a role, and the condition is strongly associated with the HLAB27 gene (Khan 2003). The term 'Axial SpA' has been used to describe the condition since diagnosis was refined by the Assessment of Spondyloarthritis International Society (ASAS) in 2009 (Rudwaleit 2009b). Axial SpA is a form of spondyloarthritis for which the predominant symptom is chronic inflammatory back pain, but radiographic sacroiliitis may or may not be present. The new classification criteria extend the diagnosis to encompass nonradiographic Axial SpA, beyond the Modified New York Crite- ria used previously (Van der Linden 1984), by which diagnosis depended upon $\mathrm{x}$-ray changes at the sacroiliac joint. The dependence on radiographic changes contributed to patients' experiencing long delays in diagnosis, with many cases remaining unrecognised (Rudwaleit 2004). The mean prevalence of AS per 10,000 is estimated at 23.8 in Europe, 16.7 in Asia, 31.9 in North America, 10.2 in Latin America and 7.4 in Africa (1.30 to 1.56 million cases in Europe; 4.63 to 4.98 million cases in Asia) (Dean 2014). Prevalence estimates based on New York or Modified New York criteria were approximately double those based on clinical criteria. One US study reported a prevalence rate of 70 cases per 10,000 while using the new ASAS criteria (Strand 2013), which may indicate the potential for higher prevalence rates, depending on the method of diagnosis applied. Prevalence is generally higher among males, with rate estimates ranging from 1.2 to seven times higher than in females (Dean 2014). However, results from a Canadian study suggest that the proportion of females is rising, with male/female ratios falling from 1.7:1 to $1.2: 1$ over a 15-year period (Haroon 2014).

Chronic symptoms, the unpredictability of flare, vulnerability to 
physical distress, the uncertain disease course and progressive impairment of physical functioning represent a significant long-term impact on lifestyle factors such as career, family and social life (Barlow 2001; Doward 2003). AS/Axial SpA is responsible for substantial direct and indirect socioeconomic costs, including work disability, which 20 years post diagnosis may be as high as $30 \%$ compared with the general population (Boonen 2001; Boonen 2002; Sieper 2002). Consequently, the condition has a significant impact on quality of life (Ward 1999; Haywood 2003). The physical and psychosocial impact of AS/Axial SpA is associated with substantial personal and societal costs that rise substantially with increasing functional limitations (Boonen 2006; Kobelt 2006; Hamilton-West 2009). Patients with a non-radiographic diagnosis experience a similar burden of disease and impact on health-related quality of life as those with radiographically confirmed Axial SpA (Rudwaleit 2009a; Kiltz 2012).

\section{Description of the intervention}

Strategies for managing AS aim to reduce symptoms of pain and stiffness, improve and/or maintain function and mobility, prevent disability, improve quality of life and prevent structural damage (Maksymowych 2006). Treatment of AS has evolved from symptomatic relief to modification of disease activity through use of biological therapies such as anti-tumour necrosis factor (TNF), which has led to reductions in morbidity and mortality (Scalapino 2003). Management of AS/Axial SpA involves a combination of pharmacological and non-pharmacological interventions. Patient education and physical exercise are the cornerstones of non-pharmacological management. Non-steroidal anti-inflammatory drugs (NSAIDs) are recommended as first-line drug treatment for patients with AS/Axial SpA with symptomatic inflammatory back pain (Braun 2011). Anti-TNF therapy may reduce disease activity in patients with highly active disease who have failed to respond to NSAIDs (Davis 2005b; Braun 2008; van der Heijde 2009), but it has not been shown to reduce radiographic progression (van der Heijde 2008; van der Heijde 2008b; van der Heijde 2009b).

Self management for people with AS/Axial SpA focuses on two key areas: medical management - such as taking medications, accompanied by a regimen of exercises, diet, etc.; and psychosocial adaptation - such as coping with anger, fear and frustration, and accommodating various new life roles in relation to jobs, family and friends (Corbin 1988; McGowan 2005). Self management has been defined as 'the individual patient ability and competence regarding the management of symptoms, treatment, physical and psychosocial consequences and the lifestyle changes inherent in living with a chronic condition' (Barlow 2000b). The core concept in self management is realisation of self efficacy, that is, confidence in oneself to carry out the required behaviour to acquire the desired goal (Bandura 1997). Education in effective self management is focused on problem solving, decision making, using resources and establishing good communication with healthcare providers (Arnold 1995; Anderson 1996; Von Korff 1997; Lorig 2003a).

In the 1970s, Kate Lorig at Stanford University was the first to develop a self management programme that was used for patients with arthritis (Brady 2003). Since that time, this programme has been applied in various chronic conditions, including osteoarthritis and rheumatoid arthritis, with evidence of improvement in self efficacy, pain, depression, disability, healthcare utilization and costs (Lorig 1993; Barlow 2000a; Lorig 2003b; Chodosh 2005; Lorig 2005; Lorig 2006; Osborne 2006; Elzen 2007). Most self management programmes have been delivered face-to-face, but now many can be delivered over the Internet (Lorig 2008).

\section{How the intervention might work}

Objectives of self management programmes include engaging in activities such as exercise to promote health, build physiological reserve and prevent adverse sequelae; empowering interaction with healthcare providers and adherence to recommended treatments and techniques; and setting goals (Osborne 2004).

\section{Why it is important to do this review}

No cure for AS/Axial SpA is known, so the main goal of treatment for individuals with this lifelong condition is to enable optimal self management. Poor self management increases the burden of disease on the individual and on healthcare resources; therefore effective disease management is essential. The overall effectiveness of self management programmes for AS/Axial SpA remains unclear. Two reviews have examined self management educational programmes but have not specifically focused on people with AS/ Axial SpA (Foster 2007; Kroon 2014); one is limited to interventions delivered by lay people (Foster 2007). Our review focuses specifically on interventions for self management delivered by any trained person for people with AS/Axial SpA.

\section{O B JE C T IVES}

To assess the benefits and harms of self management programmes for people with AS/Axial SpA.

\section{METHODS}

\section{Criteria for considering studies for this review}

\section{Types of studies}


We will include only randomised clinical trials (RCTs) and quasirandomised trials (defined as trials in which participants are allocated to study groups according to, for example, date of birth or alternate allocation) that fulfil the following criteria.

- Self management programmes versus control. Control group defined as follows: no intervention, or specific intervention (such as information or instructions on medications, exercise, information booklets or social supports) or self management programmes without a programme leader.

- Studies reporting results for pooled AS/Axial SpA populations. When data are available, we will also report populations separately.

We will include studies published in any language.

\section{Types of participants}

\section{Inclusion criteria}

Studies with participants over 18 years of age with a clinical diagnosis of AS as defined by the Modified New York criteria (Van der Linden 1984), or with a diagnosis of Axial SpA as defined by the ASAS classification criteria (Rudwaleit 2009b).

\section{Exclusion criteria}

Diagnostic criteria have not been met, or the diagnosis involves uncertainty.

\section{Types of interventions}

\section{Inclusion criteria}

Self management programme has been designed for people with AS or Axial/SpA.

Programme content must include at least one component from each of the following: biological, psychological and social management; interventions must consist of disease information about AS/Axial SpA, medication management, exercise, disease-related problem solving, cognitive symptom management, management of emotions, communication skills and use of community resources (Holman 2004).

Participants should interact with a programme leader who is a healthcare professional or a trained layperson.

Programmes may be delivered face-to-face or via the Internet, provided they meet the above criteria with direct or indirect trained support provided, as described above.

\section{Exclusion criteria}

Non-randomised studies. Studies in which interventions focus only on a specific component such as exercise, cognitive symptom management or social support.

\section{Types of outcome measures}

Effects of self management may last for a few days or for many months. We will therefore report short-term (up to six months) and longer-term (longer than six months) follow-up separately. We will measure mean improvement in score on the following outcomes (unless otherwise stated).

\section{Primary outcomes}

- Pain, e.g. Medical Outcomes Study Pain Scale or Oswestry Low Back Pain Disability Questionnaire.

- Self efficacy in symptom management/self management, e.g. General Self-Efficacy Scale or Stanford Self-Efficacy Scale (SSES).

- Positive and active engagement in life (including return to work, fulfilling roles within the family).

- Disease activity, e.g. Bath Ankylosing Spondylitis Disease Activity Index (BASDAI).

- Physical function, e.g. Bath Ankylosing Spondylitis Functional Index (BASFI).

- Number of withdrawals due to adverse events.

- Number of serious adverse events.

\section{Secondary outcomes}

- Patient's global assessment of disease activity, e.g. Bath Ankylosing Spondylitis Global assessment (BASG).

- Spinal mobility, e.g. Bath Ankylosing Spondylitis Metrology Index (BASMI).

- Health-related quality of life, e.g. Ankylosing Spondylitis Quality of Life (ASQoL), Evaluation of Ankylosing Spondylitis Quality of Life (EASIQoL), Short Form (SF)-36, EuroQoL Group Quality of Life Questionnaire (EQ5D), World Health Organization Quality of Life (WHOQoL).

- Fulfilment of response criteria, e.g. proportion of responders according to ASAS20 (improvement $\geq 20 \%$ ), ASAS40 (improvement $\geq 40 \%$ ), etc.

- Depression, e.g. Hospital Anxiety and Depression Scale.

- Fatigue, e.g. Multidimensional Assessment of Fatigue Scale.

We will report primary outcomes in a 'Summary of findings' table.

\section{Search methods for identification of studies}

See Cochrane Musculoskeletal Group (CMSG) methods used in Reviews. 


\section{Electronic searches}

We will use the search strategy developed by the Cochrane Musculoskeletal Group to search the following electronic databases for primary studies.

- The Cochrane Central Register of Controlled Trials

(CENTRAL).

- MEDLINE (Ovid) 1946 to present.

- EMBASE (Ovid) 1947 to present.

- Cumulative Index to Nursing and Allied Health Literature (CINAHL) (EBSCOHost) 1980 to present.

- PsycINFO (Ovid) 1806 to present.

- Physiotherapy Evidence Database (PEDro).

We will use the MEDLINE highly sensitive version of the RCT filter from Chapter 6 of the Cochrane Handbook for Systematic Reviews of Interventions (Lefebvre 2011). We will search for studies published in any language at any time and in any format.

We will modify the Ovid MEDLINE search strategy (Appendix 1) to suit other bibliographic databases.

\section{Searching other resources}

We will search 'grey' literature, such as reports and conference proceedings, using the System for Information on Grey Literature in Europe (SIGLE). We will search www.Clinicaltrials.gov and the World Health Organization International Clinical Trial Registry Platform (ICTRP) (www.who.int/ictrp/en) to identify trials in progress. We will contact experts in the fields of public health, rheumatology, medicine and rehabilitation to request information on unpublished and ongoing trials of self management programmes for AS.

We will also search the Database of Abstracts of Reviews of Effects (DARE) via The Cochane Library to identify potentially relevant reviews. We will screen the reference lists of reviews and primary studies to identify additional studies for inclusion in this review.

\section{Data collection and analysis}

\section{Selection of studies}

Two review authors (EM, JM) will independently assess the titles and abstracts of all retrieved trials. The same review authors will then examine the full text of papers identified as relevant to assess studies for inclusion in this review. We will resolve disagreements about eligibility and inclusion by consulting with a third review author (SS).

\section{Data extraction and management}

SS and JM will independently read and extract data from included studies using a form based on predefined outcome measures. We will contact study authors to request information on missing data or further information about the trial. We will systematically record information on study design, participants, interventions, outcomes, methods, results and study withdrawals in 'Characteristics of included studies' tables.

We will resolve disagreements by consulting with a third review author (PM).

When possible, review authors will report with a consistent direction of effect (i.e. favourable outcome to left of line of no effect).

\section{Assessment of risk of bias in included studies}

SS and JM will independently assess each included study for risk of bias using the criteria described in the Cochrane Handbook for Systematic Reviews of Interventions (Higgins 2011). We will rate risk of bias as high, low or unclear across the domains below. We will resolve disagreements by consulting with a third review author and will tabulate results in a 'Risk of bias' table.

\section{Random sequence generation}

- Low risk of bias: adequate sequence generation (e.g. random number tables, coin toss, drawing lots, dice throw).

- High risk of bias: inadequate sequence generation using a non-random method of allocation (e.g. date of birth, hospital admission date, clinic number).

- Unclear risk of bias: information on sequence generation not given or unclear.

\section{Allocation concealment}

- Low risk of bias: adequate concealment (e.g. central allocation method such as telephone or Web-based randomisation, sealed opaque envelopes).

- High risk of bias: inadequate concealment of allocation (e.g. open list of numbers, envelopes without concealed contents, dates of birth).

- Unclear risk of bias: information on allocation of randomisation not given or unclear.

\section{Blinding of participants and personnel}

- Low risk of bias: adequate when study participants and personnel are blinded to allocated interventions, or when review authors judge that study outcomes will not be influenced by lack of blinding.

- High risk of bias: inadequate when study outcomes are likely to be influenced by lack of or incomplete blinding.

- Unclear risk of bias: information on blinding of participants and personnel not given or unclear. 


\section{Blinding of outcome assessment}

- Low risk of bias: adequate when study participants and personnel are blinded to outcome assessment, or when review authors judge that outcome measures will not be influenced by lack of blinding.

- High risk of bias: inadequate when measurement of outcomes is not blinded and may be influenced by lack of blinding.

- Unclear risk of bias: information on blinding of outcome assessment not given or unclear.

\section{Incomplete outcome data}

- Low risk of bias: adequate (e.g. no missing data, missing data unrelated to true outcome (e.g. survival data), balanced across study groups, reasons for missing data similar across groups).

- High risk of bias: inadequate (e.g. missing data may be related to true outcome (missing not at random), reasons for missing data or missing proportions differ between groups, imputation is inappropriate (e.g. high proportion of data imputed using last observation carried forward)).

- Unclear risk of bias: information on incomplete outcome data not given or unclear.

\section{Selective reporting}

- Low risk of bias: adequate (e.g. clear that all prespecified or expected study outcomes have been reported consistently).

- High risk of bias: inadequate (e.g. not all prespecified outcomes reported, primary outcomes reported that were not prespecified, outcomes reported using methods not prespecified).

- Unclear risk of bias: information on outcome reporting not given or unclear.

\section{Other bias}

- Low risk of bias: adequate when no other sources of bias are identified.

- High risk of bias: inadequate when other important sources of bias are identified, such as inappropriate study design.

- Unclear risk of bias: insufficient information for evaluation of risk of other bias.

\section{Measures of treatment effect}

\section{Binary data}

For binary data, risk ratio (RR), Peto odds ratio (for rare events $<10 \%$ ) and $95 \%$ confidence intervals (CIs) will be estimated according to intention-to-treat principles. For pooled data, the fixed-effect model will be preferred, but the random-effects model will be used when heterogeneity is present.

\section{Continuous data}

Skewed data: Continuous data on clinical and social outcomes often are not normally distributed. To avoid the pitfall of applying parametric tests to non-parametric data, we will apply the following standards to all data before inclusion: (1) Standard deviations and means must be reported in the paper or must be obtainable from study authors; (2) when a scale starts from a finite number (such as zero), the standard deviation, when multiplied by two, must be less than the mean (as otherwise, the mean is unlikely to be an appropriate measure of the centre of the distributions) (Altman 1996). Endpoint scores on scales often have finite start and end points, and this rule can be applied to them. When sample sizes are thought to be too small, we will consider non-parametric and more exact statistical procedures. We will seek assistance from the CMSG statistician, should the need arise.

Summary statistic: For continuously distributed outcomes, we will calculate the mean difference (WMD) when the same scale is used in a similar manner across studies; otherwise, we will use the standardised mean difference (SMD). We will use a fixedeffect model in the first instance but the random-effects model to investigate the sensitivity of results to choice of statistical method.

\section{Unit of analysis issues}

\section{Cross-over trials}

We will use only data from the first pre-cross-over phase to minimise potential bias from carry-over effects.

\section{Cluster trials}

We will analyse these in accordance with methods described in Section 16.3 of the Cochrane Handbook for Systematic Reviews of Interventions (Higgins 2011), using average cluster size and an estimate of the intraclass correlation co-efficient (ICC) to adjust sample sizes to the 'effective sample size'. When an estimate of the ICC is not available from trial information, we will use an estimate from a similar trial or from a trial with a similar population. We will combine single RCTs with cluster RCTs if we consider designs and interventions sufficiently similar, and if the effect of the intervention is not likely to be influenced by the method of randomisation.

\section{Multiple-arm trials}

For trials with more than two arms, we will describe all study groups in the 'Characteristics of included studies' table, but we will include in the analysis only intervention groups that meet our 
review criteria. When the variance of the difference between intervention and comparator is not reported, we will calculate this from the variances of all trial arms. When a study includes multiple relevant treatment arms, we will combine groups to perform a single pairwise comparison (Higgins 2011). If this prevents identification of potential heterogeneity, we will analyse each group separately against a common control group. However, to ensure that a common control group is not included multiple times in a metaanalysis that includes several interventions from the same trial, we will proportionately reduce control group data (Higgins 2011). For example, in a study with two interventions and a single control group, we will halve the numbers of participants and events in the control group. When studies report only differences between treatment groups, as opposed to mean effects for each group, we will analyse data using the generic inverse variance function.

\section{Dealing with missing data}

We will contact all authors of included studies to request unreported data such as missing outcomes, missing data, means or SDs. We will note differential dropout between study groups and reasons for withdrawal. When a particular outcome includes substantial loss to follow-up (50\%), we will report this in the text and will mark the data with an asterisk. We will note differential missing data and reasons for missing data, when reported. We will use intention-to-treat (ITT) principles for data analysis and will not impute missing data. When studies report per-protocol data (i.e. only those who completed the study), we will contact study authors to request unreported data on all study participants. We will describe missing data in the 'Risk of bias' table and will discuss in the text the influence of missing data on study outcomes. If trials are sufficient, we will use sensitivity analyses to determine the resistance of our results to the effects of missing data.

\section{Assessment of heterogeneity}

We will assess heterogeneity by using the $\mathrm{Chi}^{2}$ test in conjunction with the $\mathrm{I}^{2}$ statistic. We will set significance for the $\mathrm{Chi}^{2}$ test at $\mathrm{P}$ value $\leq 0.10$ because of the low power of this test (Higgins 2011). We will keep the cut point for $\mathrm{I}^{2}$ at $50 \%$. When significant heterogeneity is present, we will attempt to explain differences based on clinical characteristics of included studies.

\section{Assessment of reporting biases}

We will compare data from published and unpublished studies as a direct test of publication bias. If we identify a sufficient number of studies (approximately $\geq 10$ ), we will explore potential reporting biases and small study effects by using Egger's method (Egger 1997 ) to test for asymmetry in funnel plots.

\section{Data synthesis}

We will included in meta-analyses studies in which study designs, interventions and outcomes are similar. When we identify substantial heterogeneity, we will report outcomes narratively in the text. It is likely that studies on self management programmes for AS will vary by population, design and outcomes, so we will use a random-effects model for pooled analyses. However, when few studies are found (as may be the case with this review) or when effects of the intervention are not randomly distributed across studies (e.g. with publication bias), random-effects model estimates may be unreliable. To resolve uncertainty over model choice, we will (1) pool data using meta-analyses only when studies appear sufficiently similar, and (2) compare pooled data estimates from both random-effects and fixed-effect models, while reporting both in the text. We will report the mean effect estimate and the confidence interval (CI) around the estimate for both models, noting that the CI for the random-effects model is not an estimate of heterogeneity. For dichotomous outcomes, we will sum sample sizes and events across groups, and for continuous outcomes, we will combine means and standard deviations using the formula described in Chapter 7 of the Cochrane Handbook for Systematic Reviews of Interventions (Section 7.7.3.8 Higgins 2011). We will synthesise and report dichotomous and continuous data separately for a given outcome, should the need arise. When both end-ofstudy differences and changes from baseline are reported, we will analyse these separately. We will perform these analyses using Review Manager 5.2.

\section{Subgroup analysis and investigation of heterogeneity}

\section{Subgroup analyses}

When sample sizes of intervention groups permit, we will conduct subgroup analyses of the following.

- Disease classification: AS versus Axial SpA.

- Intervention type: We will ask an independent group of researchers to classify the interventions while remaining blinded to treatment effects.

\section{Investigation of heterogeneity}

If numbers of studies are sufficient, we will manage potential sources of heterogeneity as follows.

- Check data integrity, including measures of effect and units of analysis.

- Explore the impact of subgroups (e.g. small vs large studies).

- Exclude outliers when the reason for exclusion is clear. We will inspect the graph and will iteratively remove outlying studies to determine whether homogeneity is restored.

We will fully discuss and report our decisions in the review. 


\section{Sensitivity analysis}

We will perform sensitivity analysis by excluding studies with the following.

- High $\mathrm{I}^{2}(\geq 50 \%)$.

- Inadequate allocation concealment.

- Low attrition rates.

We will compare results with and without data from unpublished studies.

\section{'Summary of findings' table}

We will report primary outcomes of the review in a 'Summary of findings' table (self efficacy, positive and active engagement in life, disease activity, global well-being, physical function, health-related quality of life and adverse events). The 'Summary of findings' table will rate evidence related to each outcome according to the GRADE (Grades of Recommendation, Assessment, Development and Evaluation) criteria (Schunemann 2011).

We will express pooled SMDs as mean differences by multiplying each SMD by a relevant trial control group baseline standard deviation. In the comments column, we will report the absolute percentage change and the relative percentage change, and for out- comes with statistically significant differences between groups, we will also report the number needed to treat for an additional beneficial outcome (NNTB).

For dichotomous outcomes, we will calculate the following: absolute risk difference expressed as a percentage; relative percentage change (risk ratio -1) expressed as a percentage; NNTB from the control group event rate and risk ratio obtained with the Visual Rx NNTB calculator (Cates 2013).

For continuous outcomes, we will calculate the following: absolute risk difference (mean difference divided by the scale) expressed as a percentage; and relative difference (change or mean difference divided by relevant control group baseline mean). We will use the Wells calculator to obtain the NNTB for continuous measures (available at the CMSG Editorial office; http://musculoskeletal.cochrane.org/).

\section{ACKNOWLEDGEMEN T S}

The review authors would like to thank the Cochrane Musculoskeletal Group for valuable comments on the protocol and for support provided during development of this protocol.

\section{REFEREN CES}

\section{Additional references}

\section{Altman 1996}

Altman DG, Bland JM. Detecting skewness from summary information. British Medical Journal 1996;313:1200.

Anderson 1996

Anderson RM, Funnell MM, Arnold MS. Practical psychology for diabetes clinicians. In: Rubin B, Anderson B editor(s). Using the Empowerment Approach to Help Patients Change Behaviour. 2nd Edition. Alexandria, VA: American Diabetes Association, 1996.

\section{Arnold 1995}

Arnold MS, Butler RM, Anderson RM, Funnell MM, Feste C. Guidelines for facilitating a patient empowerment. The Diabetes Educators 1995;21(4):308-12.

Bandura 1997

Bandura A. Self-efficacy: The Exercise of Control. New York, NY: Worth Publishers, 1997.

Barlow 2000a

Barlow JH, Turner AP, Wright CC. A randomized controlled study of the arthritis self-management programme in the UK. Health Education Research 2000a;15(6):665-80.

\section{Barlow 2000b}

Barlow J, Williams B, Wright C. Patient education for people with arthritis in rural communities: the UK experience. Patient Education and Counselling 2000;1451: $1-10$.

\section{Barlow 2001}

Barlow JH, Wright CC, Williams B, Keat A. Work disability among people with ankylosing spondylitis. Arthritis and Rheumatism 2001;45:424-9.

Boonen 2001

Boonen A, Chorus A, Miedema H, Van der Heijde D. Withdrawal from labour force due to work disability in patients with ankylosing spondylitis. Annals of the Rheumatic Diseases 2001;60(11):1033-40.

Boonen 2002

Boonen A, van der Heijde D, Landewe R, Spoorenberg A, Schouten H, Rutten-van Mölken M, et al. Work status and productivity costs due to ankylosing spondylitis: comparison of three European countries. Annals of the Rheumatic Diseases 2002;61:429-37.

\section{Boonen 2006}

Boonen A, van der Linden SM. The burden of ankylosing spondylitis. Journal of Rheumatology Supplement 2006;78: 4-11.

\section{Brady 2003}

Brady TJ, Kruger J, Helmick CG, Callahan LF, Boutaugh ML. Intervention programs for arthritis and other rheumatic diseases. Health Education \& Behavior 2003;30(1):44-63.

\section{Braun 2008}

Braun J, Deodhar A, Dijkmans B, Geusens P, Sieper J, Williamson P, et al. Efficacy and safety of infliximab in 
patients with ankylosing spondylitis over a two-year period. Arthritis and Rheumatism 2008;59:1270-8.

Braun 2011

Braun J, van den Berg R, Baraliakos X, Boehm H, BurgosVargas R, Collantes-Estevez E, et al. 2010 update of the ASAS/EULAR recommendations for the management of ankylosing spondylitis. Annals of the Rheumatic Diseases 2011;70:896-904.

Cates 2013

Cates C. Visual Rx 2.0 NNT Calculator. www.nntonline.net 2013.

\section{Chodosh 2005}

Chodosh J, Morton SC, Mojica W, Maglione M, Suttorp MJ, Hilton L, et al. Meta-analysis: chronic disease selfmanagement programs for older adults. Annals of Internal Medicine 2005;143:427-38.

\section{Corbin 1988}

Corbin J, Strauss A. Unending Work and Care: Managing Chronic Illness at Home. 1st Edition. San Francisco, CA: Jossey-Bass, 1988.

Davis 2005

Davis JC Jr. Ankylosing spondylitis. Arthritis and Allied Conditions. Baltimore, MD: Lippincott Williams \& Wilkins, 2005.

Davis 2005b

Davis J, van der Heijde DM, Braun J, Dougados M, Cush J, Clegg D, et al. Sustained durability and tolerability of etanercept in ankylosing spondylitis for 96 weeks. Annals of the Rheumatic Diseases 2005;64:1557-62.

\section{Dean 2014}

Dean LE, Jones GT, MacDonald AG, Downham C, Sturrock RD, Macfarlane GJ. Global prevalence of ankylosing spondylitis. Rheumatology 2014;53:650-7.

\section{Doward 2003}

Doward LC, Spoorenberg A, Cook SA. Development of the ASQoL: a quality of life instrument specific to ankylosing spondylitis. Annals of the Rheumatic Diseases 2003;62:20-6.

\section{Egger 1997}

Egger M, Davey-Smith G, Schneider M, Minder C. Bias in meta-analysis detected by a simple, graphical test. Bristish Medical Journal 1997;315(7109):629-34.

\section{Elzen 2007}

Elzen H, Slaets JP, Snijders TA, Steverink N. Evaluation of the chronic disease self-management program (CDSMP) among chronically ill older people in the Netherlands. Social Science \& Medicine 2007;64(9):1832-41.

Foster 2007

Foster G, Taylor SJC, Eldridge S, Ramsay J, Griffiths CJ. Self-management education programmes by lay leaders for people with chronic conditions. Cochrane Database of Systematic Reviews 2007, Issue 4. Art. No.: CD005108. DOI: 10.1002/14651858.CD005108.pub2. [DOI: 10.1002/14651858.CD005108.pub2]

\section{Hamilton-West 2009}

Hamilton-West K, Quine L. Living with ankylosing spondylitis. The patient's perspective. Journal of Health Psychology 2009;14:820-30.

\section{Haroon 2014}

Haroon NN, Paterson JM, Li P, Haroon N. Increasing proportion of female patients with ankylosing spondylitis: a population-based study of trends in the incidence and prevalence of AS. BMJ Open 2014;4:e006634.

\section{Haywood 2003}

Haywood K, Garratt A, Dziedzic K, Dawes P. Patient centred assessment of ankylosing spondylitis-specific health related quality of life: evaluation of the Patients Generated Index. The Journal of Rheumatology 2003;30:764-73.

\section{Higgins 2011}

Higgins JPT, Green S (editors). Cochrane Handbook for Systematic Reviews of Interventions Version 5.1.0 [updated March 2011]. The Cochrane Collaboration, 2011. www.cochrane-handbook.org.

\section{Holman 2004}

Holman H, Lorig K. Patient self-management: a key to effectiveness and efficiency in care of chronic disease. Public Health Reports 2004;119(3):239-3.

Khan 2003

Khan MA. Clinical features of ankylosing spondylitis. In: Hochberg MC, Silman AJ, Smolen JS, Weinblatt ME, Weisman MH editor(s). Rheumatology. 3rd Edition. London: Mosby, 2003:1161-81.

\section{Kiltz 2012}

Kiltz U, Baraliakos X, Karakostas P, Igelmann M, Kalthoff L, Klink C, et al. Do patients with non-radiographic axial spondylarthritis differ from patients with ankylosing spondylitis?. Arthritis Care and Research 2012;64:1415-22.

\section{Kobelt 2006}

Kobelt G, Andlin-Sobocki P, Maksymowych WP. Cost and quality of life of patients with ankylosing spondylitis in Canada. Journal of Rheumatology 2006;33:289-95.

\section{Kroon 2014}

Kroon FPB, van der Burg LRA, Buchbinder R, Osborne RH, Johnston RV, Pitt V. Self-management education programmes for osteoarthritis. Cochrane Database of Systematic Reviews 2014, Issue 1. Art. No.: CD008963. DOI: 10.1002/14651858.CD008963.pub2.

\section{Lefebvre 2011}

Lefebvre C, Manheimer E, Glanville J. Chapter 6: Searching for studies. In: Higgins JPT, Green S editor(s). Cochrane Handbook for Systematic Reviews of Interventions Version 5.1.0 [updated March 2011]. The Cochrane Collaboration, 2011. www.cochrane-handbook.org.

\section{Lorig 1993}

Lorig K, Holman H. Arthritis self-management studies: a twelve-year review. Health Education \& Behavior 1993;20 (DOI: 10.1177/109019819302000104):17-28. 


\section{Lorig 2003a}

Lorig K, Holman H. Self-management education: history, definition, outcomes, and mechanisms. Annals of Behavioral Medicine 2003a;26(1):1-7.

Lorig 2003b

Lorig K, Ritter PL, Gonzalez VM. Hispanic chronic disease self-management: a randomized community-based outcome trial. Nursing Research 2003b;52(6):361-9.

Lorig 2005

Lorig K, Ritter PL, Plant K. A disease-specific self-help program compared with a generalized chronic disease selfhelp program for arthritis patients. Arthritis \& Rheumatism 2005;53(7):950-7.

Lorig 2006

Lorig K, Ritter PL, Laurent DD, Plant K. Internet-based chronic disease self-management: a randomized trial. Medical Care 2006;44(11):964-71.

Lorig 2008

Lorig LR, Ritter PL, Dost A, Plant K, Laurent DD, Mcneil I. The expert patients programme online, a 1-year study of an internet-based self-management programme for people with long-term conditions. Chronic Illness 2008;4:247-56. [DOI: $10.1177 / 1742395308098886$ ]

\section{Maksymowych 2006}

Maksymowych WP. Treatment. In: Weisman MH, Van der Heijde D, Reville JD editor(s). Ankylosing Spondylitis and the Spondyloarthropathies. Philadelphia: Mosby/Elsevier, c2006:154-89.

\section{McGowan 2005}

McGowan P. Self-management: a background paper. New Perspectives: International Conference on Patient SelfManagement. Victoria, British Columbia, Canada: Center on Aging, 2005:1-10.

Osborne 2004

Osborne RH, Spinks JM, Wicks IP. Patient education and self-management programme in arthritis. The Medical Journal of Australia 2004;180(5 suppl):S23-6.

Osborne 2006

Osborne RH, Buchbinder R, Ackerman IN. Can a diseasespecific education program augment self-management skills and improve health-related quality of life in people with hip or knee osteoarthritis?. BMC Musculoskeletal Disorders 2006;7:90.

\section{Rudwaleit 2004}

Rudwaleit M, van der Heijde D, Khan MA, Braun J, Sieper J. How to diagnose axial spondyloarthritis early. Annals of the Rheumatic Diseases 2004;63:535-43. [DOI: 10.1136/ ard.2003.011247]

\section{Rudwaleit 2009a}

Rudwaleit M, Haibel H, Baraliakos X, Listing J, MarkerHermann E, Zeidler $\mathrm{H}$, et al. The early disease stage in axial spondyloarthritis: results from the German Spondyloarthritis Inception Cohort. Arthritis and Rheumatism 2009;60:717-27.

\section{Rudwaleit 2009b}

Rudwaleit M, van der Heijde D, Landewe R, Listing J, Akkoc N, Brandt J, et al. The development of Assessment of SpondyloArthritis International Society classification criteria for axial spondyloarthritis (part II): validation and final selection. Annals of the Rheumatic Diseases 2009;68: 777-83. [DOI: 10.1136/ard.2009.108233]

\section{Scalapino 2003}

Scalapino KJ, Davis JC Jr. The treatment of ankylosing spondylitis. Clinical and Experimental Medicine 2003;2: 159-65.

\section{Schunemann 2011}

Schünemann HJ, Oxman AD, Vist GE, Higgins JPT, Deeks JJ, Glasziou P, et al. Chapter 12: Interpreting results and drawing conclusions. In: Higgins JPT, Green S editor(s). Cochrane Handbook for Systematic Reviews of Interventions Version 5.1.0 [updated March 2011]. The Cochrane Collaboration, www.cochrane-handbook.org, 2011.

\section{Sieper 2002}

Sieper J, Braun J, Boonen A, Zink A. Ankylosing spondylitis: an overview. Annals of the Rheumatic Diseases 2002;61(Suppl III):iii8-iii18.

\section{Strand 2013}

Strand V, Rao SA, Shillington AC, Cifaldi MA, Mcguire M, Ruderman EM. Prevalence of axial spondyloarthritis in United States rheumatology practices: assessment of SpondyloArthritis International Society criteria versus rheumatology expert clinical diagnosis. Arthritis Care and Research 2013;65(8):1299-306.

\section{van der Heijde 2008}

van der Heijde D, Landewé R, Einstein S, Ory P, Vosse $\mathrm{D}, \mathrm{Ni} \mathrm{L}$, et al. Radiographic progression of ankylosing spondylitis after up to two years of treatment with etanercept. Arthritis and Rheumatism 2008;58:1324-31.

\section{van der Heijde 2008b}

van der Heijde D, Landewe R, Baraliakos X, Houben H, van Tubergen A, Williamson P, et al. Radiographic findings following two years of infliximab therapy in patients with ankylosing spondylitis. Arthritis and Rheumatism 2008;58: 3063-70.

van der Heijde 2009 van der Heijde D, Schiff MH, Sieper J, Kivitz AJ, Wong $\mathrm{RL}$, Kupper $\mathrm{H}$, et al. Adalimumab effectiveness for the treatment of ankylosing spondylitis is maintained for up to 2 years: long-term results from the ATLAS trial. Annals of the Rheumatic Diseases 2009;68:922-9.

van der Heijde 2009b

van der Heijde D, Salonen D, Weissman BN, Landewé R, Maksymowych WP, Kupper H, et al. Assessment of radiographic progression in the spines of patients with ankylosing spondylitis treated with adalimumab for up to 2 years. Arthritis Research and Therapy 2009;11:R127.

\section{Van der Linden 1984}

Van der Linden S, Valkeuburg HA, Cats A. Evaluation of diagnostic criteria for ankylosing spondylitis: a proposal 
for modification of the New York criteria. Arthritis and

Rheumatism 1984;27(4):361-8.

\section{Von Korff 1997}

Von Korff M, Gruman J, Schaefer J, Curry SJ, Wagner EH.

Collaborative management of chronic illness. Annals of

Internal Medicine 1997;127:1097-102.

Ward 1999

Ward M. Health-related quality of life in ankylosing

spondylitis: a survey of 175 patients. Arthritis Care and

Research 1999;12:247-55.

* Indicates the major publication for the study

\section{A P P E N D I CES}

\section{Appendix I. Search strategy}

Database: Ovid MEDLINE(R) In-Process \& Other Non-Indexed Citations and Ovid MEDLINE(R) $<1946$ to Present $>$ Search Strategy:

1 Spondylitis, Ankylosing/ (11620)

2 Spondylarthritis/ (815)

3 (axial adj2 spondylarthritis).tw. (16)

4 (axial adj2 spa).tw. (151)

5 (ankylos\$ or spondyl\$).tw. (30174)

6 (bekhterev\$ or bechterew\$).tw. (665)

7 (Marie adj struempell\$).tw. (6)

8 or/1-7 (33478)

9 exp Self Care/ (39952)

10 ((self or symptom\$) adj (care or help or manag\$ or directed or monitor $\$$ or efficacy or admin $\$)$ ).tw. (72002)

11 Patient Education as Topic/ (69613)

12 ((health or patient\$) adj2 (educat\$ or information)).tw. (79427)

13 exp Consumer Participation/ (31753)

14 ((patient\$ or consumer\$) adj part\$).tw. (16172)

15 "power (psychology)"/ (9933)

16 empower\$.tw. (12584)

17 Holistic Health/ (6985)

18 (holistic or wholistic).tw. (11875)

19 exp Rehabilitation/ (147043)

20 rehab\$.tw. (107810)

21 "Activities of Daily Living"/ (49414)

22 (activit\$ adj2 daily adj living).tw. (16033)

23 social support/ (50659)

24 (social adj (support or network\$)).tw. (27981)

25 (support adj system\$).tw. (8741)

26 exp Adaptation, Psychological/ (101323)

27 (psychologic\$ adj (adjust\$ or adapt\$)).tw. (2473)

28 (cope or copes or coping).tw. (50737)

29 exp Behavior Therapy/ (51924)

30 (adapt $\$$ adj behav\$).tw. (3007)

Self management programme for ankylosing spondylitis (Protocol)

Copyright (־ 2015 The Cochrane Collaboration. Published by John Wiley \& Sons, Ltd. 
31 (behav\$ adj (therap\$ or intervention\$)).tw. (17332)

32 or/9-31 (691720)

338 and 32 (1269)

34 randomized controlled trial.pt. (370699)

35 controlled clinical trial.pt. (88158)

36 randomized.ab. (290757)

37 placebo.ab. (152961)

38 clinical trials as topic.sh. (169353)

39 randomly.ab. (210816)

40 trial.ti. (125012)

41 or/34-40 (896351)

42 exp animals/ not humans.sh. (3923244)

4341 not 42 (827154)

4433 and 43 (138)

\section{WHAT'S NEW}

\begin{tabular}{|c|c|c|}
\hline Date & Event & Description \\
\hline 13 April 2015 & New citation required and minor changes & Protocol rewritten with new author team \\
\hline
\end{tabular}

\section{H I S T O R Y}

Protocol first published: Issue 1, 2008

\begin{tabular}{lll}
\hline Date & Event & Description \\
\hline 25 July 2008 & Amended & $\begin{array}{l}\text { Converted to new review format } \\
\text { CMSG ID: C150-P }\end{array}$ \\
\hline
\end{tabular}

\section{CONTRIBUTIONSOFAUTHORS}

Sally Spencer - will formulate title, background, objectives and search criteria.

Jane Martindale - will formulate background, objectives, outcome measures and search criteria.

Elizabeth MacPhie - will formulate background, objectives, outcome measures and search criteria.

Dr. Paul Montgomery - will develop the methods section and will edit the protocol. 
DECLARATIONSOF INTEREST

None. 